Sādhanā Vol. 39, Part 5, October 2014, pp. 1071-1094. (C) Indian Academy of Sciences

\title{
An implicit non-staggered Cartesian grid method for incompressible viscous flows in complex geometries
}

\author{
A K DE \\ Department of Mechanical Engineering, Indian Institute of Technology Guwahati, \\ Guwahati 781 039, India \\ e-mail: akd@iitg.ernet.in
}

MS received 9 January 2012; revised 5 May 2014; accepted 14 May 2014

\begin{abstract}
A discrete forcing based Cartesian grid method is presented. The nonstaggered arrangement of velocity and pressure is considered. The pressure gradient in localized discrete form is added separately with the velocity making them explicitly coupled. The governing equation is time-integrated implicitly with both linearized and non-linear forms are investigated. Both linear and bi-linear reconstruction techniques are tested for extrapolation of velocity near a complex boundary. The present method is tested for vortical flow in an inclined cavity, flow past circular and inclined square cylinder. Both homogeneous and non-homogeneous Dirichlet forcing problems are tested. The parallelized version of the method is applied to $2 \mathrm{D}$-to-3D transitional flow behind a single and multiple circular cylinders. The present numerical results compare well with the previously documented results.
\end{abstract}

Keywords. Immersed boundary; non-staggered; implicit; viscous flow.

\section{Introduction}

One of the major issues of numerical computation of fluid flow is the ability of handling complex geometries that appear in numerous situations ranging from practical flows to problems of fundamental importance. Though, a number of techniques, such as the coordinate transformation, non-conforming chimera grid technique have been demonstrated in the structured grid domain, each of them have their own issues that limit their direct application. It is obvious that if any arbitrary domain is handled with Cartesian grids the computational efficiency, in terms of accuracy achievable and the cost of computation, is enhanced significantly. This motivation has led to the idea of Cartesian grid method which is more popularly known as the immersed boundary technique. In this method, a complex surface is kept immersed in non-conforming Cartesian grids and the fluid flow equations are solved satisfying all the physical constraints.

The immersed boundary method can be broadly classified into two groups; the continuous forcing approach and the direct forcing technique. Peskin (1972) first introduced the idea of 
using Cartesian grids for flow simulation in heart valves where he modelled the force field on a surface by a distributed function. Since then a number variants proposing different distribution functions for elastic boundaries (Saiki \& Biringen 1996; Lai \& Peskin 2000; Zhu \& Peskin 2003) and rigid surfaces (Goldstein et al 1993; Angot et al 1999; Iaccarino \& Verzicco 2003) have appeared. Arthurs et al (1998), Bottino (1998) and McQueen \& Peskin (1997) applied this technique in biological flows. Successful application of this technique can be found in a variety of flows as wide as locomotion of aquatic animals (Fauci 1990), flow of suspensions (Fogelson \& Peskin 1988) and multi-phase flows (Unverdi \& Tryggvason 1992). However, this method inherently demands smoothing of the distributed function for rigid surfaces which is not suitable for flows with small scale changes, especially at high Reynolds numbers.

In the second category, a number of discrete points are selected on the immersed surface where the boundary conditions are forced to satisfy directly. Mohd-Yosuf (1997) and later Kim et al (2001) introduced a forcing term in the Navier-Stokes equations which enforces the noslip boundary condition on a solid surface while keeping the fluid domain unchanged. As the physical constraints are directly (and thus more accurately) forced on the boundaries, this technique is more suitable for flows that involve complex evolution. However, implementation of the boundary forcing has a strong relationship (Mittal \& Iaccarino 2005) with the solution technique, though a consistent boundary treatment does not restrict the time-step of the marching algorithm.

In the discrete direct forcing approach, usually a number of closely connected fluid points are extrapolated for the evaluation of the forcing term. Thus, stability and convergence behaviour of the method principally depends on the extrapolation technique incorporated. Both linear as well as bi-linear interpolation procedures were successfully employed (Kim et al 2001; Fadlun et al 2000; Tseng \& Ferziger 2003; Verzicco et al 2000; Ye et al 1999) for the boundary forcing, though Majumdar et al (2001) and later Tseng \& Ferziger (2003) found no appreciable improvement by using higher-order interpolation technique in both laminar as well as turbulent flows. Among several modified methods, Tau (1994), Tucker \& Pan (2000) and Kirkpatrick et al (2003) proposed the cut-cell method where the arbitrary intersected boundary cells are treated with their true shapes. For more complicated shapes this technique requires generation of the surface, determination of the arbitrary boundary cells and thus primarily it operates on a hybrid mesh. Attempts have also been made with the complex boundaries immersed in curvilinear structured grids (Ge \& Sotiropoulos 2007; Borazjani et al 2008), unstructured mesh (Xia et al 2009; Denaro \& Sarghini 2002) and overlapping chimera-type grids (Tai et al 2005). However, in such forms the advantages of Cartesian grid computations are partly lost because of algorithmic complexity, advanced data structure and fall in accuracy otherwise achievable. On the same note this method has received wide acceptance because of its prospect of handling complex geometry without bringing in new difficulties. For instance, applications including fluid structure interaction (Huang \& Sung 2009; Gilmanov \& Acharya 2008), large-eddy simulation of turbulent flows (Li \& Wang 2004; Tyagi \& Acharya 2005; Balaras 2004) and two-phase flows (Yang \& Stern 2009; Berthelsen \& Ytrehus 2007) have emphasized this aspect.

A major concern in flow calculations using non-staggered arrangement of variables is the weak coupling between velocity and pressure which often leads to numerical wiggles. To alleviate this issue, use of staggered grid is generally preferred. However, over the years, use of non-staggered arrangement has found its application where an adequate amount of numerical dissipation (Rhie \& Chow 1983; der Wijngaart 1990; Armfield 1991; Barton \& Kirby 2000) is added to damp out the oscillations. The present paper treats this issue in a different way: the discrete pressure gradient which is realized to drive the flow locally, is added with the velocity computed without the effects of pressure and thereby explicitly achieving a strong coupling between them. The 
solution strategy lends its idea from the projection method, though it focusses on stable implicit treatment of the variables - both in linearized and non-linear forms. The solution of the pressure Poisson equation, the heaviest task in incompressible flows, is briefly reviewed in the light of pre-conditioned conjugate gradient method when applied to the immersed boundary method. The entire solution procedure is parallelized to facilitate its use in large three-dimensional flows involving complex geometries. As the present work does not address the accuracy and resolution properties of this method, the test cases are limited to moderate Reynolds number which includes laminar and 2D-to-3D transitional flows.

The paper is organized as follows. Section 2 briefly describes the governing equations followed by detailed discussion on the present projection method, implicit treatment of the variables, the immersed boundary technique, linear solvers and the vectorization method in section 3 . In section 4 , results from a number of internal and external flow test cases involving complex boundaries are reported that measure various aspects of the present technique.

\section{Governing equations}

Incompressible viscous flows are governed by the continuity and momentum equations, written below in normalized form by using suitable length $(L)$, velocity $(U)$ and time $(L / U)$ scales

$$
\begin{aligned}
\frac{\partial u_{i}}{\partial x_{i}} & =0 \\
\frac{\partial u_{i}}{\partial t}+\frac{\partial\left(u_{i} u_{j}\right)}{\partial x_{j}} & =-\frac{\partial p}{\partial x_{i}}+\frac{1}{R e} \frac{\partial^{2} u_{i}}{\partial x_{j} \partial x_{j}},
\end{aligned}
$$

where Reynolds number is defined as $R e=U L / \nu$.

\section{Numerical details}

Using the finite volume approximations, Eqs. (1-2) can be written in terms of approximate integrals for a cell denoted by its center $P$, faces $f$ and volume $V_{P}$

$$
\begin{gathered}
\sum_{f} F_{f}^{n+1}=0 \\
\frac{u_{i, P}^{n+1}-u_{i, P}^{n}}{\Delta t} V_{P}+\sum_{f}\left(c_{1} F_{f}^{n+1} u_{i, f}^{n+1}+c_{2} F_{f}^{n} u_{i, f}^{n}+c_{3} F_{f}^{n-1} u_{i, f}^{n-1}\right)= \\
-\frac{1}{2} \sum_{f}\left(p_{f}^{n+1}+p_{f}^{n}\right) S_{f, i}+\frac{1}{2 R e} \sum_{f}\left(F_{d f i}^{n+1}+F_{d f i}^{n}\right),
\end{gathered}
$$

where $c_{1}=c_{2}=1 / 2, c_{3}=0$ and $c_{1}=0, c_{2}=3 / 2, c_{3}=-1 / 2$ correspond to the $2^{\text {nd }}$-order implicit Crank-Nicolson (CN) and semi-implicit Adams-Bashforth-Crank-Nicolson (ABCN) time integration schemes, respectively. Note that diffusion flux of a scalar $\phi$ is given as

$$
\int_{V} \frac{\partial^{2} \phi}{\partial x_{j} \partial x_{j}} d V=\int_{V} \nabla \cdot \nabla \phi d V=\int_{S} \nabla \phi \cdot \boldsymbol{d} \boldsymbol{S}=\sum_{f}(\nabla \phi \cdot S)_{f}=\sum_{f} F_{d \phi i} .
$$




\subsection{Velocity pressure coupling}

As the pressure field is not known at the $(n+1)^{\text {th }}$ time-level, first a provisional velocity field $\left(u_{i}^{*}\right)$ is predicted excluding the pressure term with mass flux taken as the latest available one

$$
\begin{array}{r}
\frac{u_{i, P}^{*}-u_{i, P}^{n}}{\Delta t} V_{P}+\sum_{f}\left(c_{1} F_{f}^{n+1, l} u_{i, f}^{*}+c_{2} F_{f}^{n} u_{i, f}^{n}+c_{3} F_{f}^{n-1} u_{i, f}^{n-1}\right)= \\
\frac{1}{2 \operatorname{Re}} \sum_{f}\left(F_{d f i}^{*}+F_{d f i}^{n}\right)
\end{array}
$$

The face velocity is then calculated by adding a pressure gradient with the provisional velocity linearly interpolated at the face

$$
\boldsymbol{u}_{f}=L\left(\boldsymbol{u}_{\boldsymbol{P}}^{*}, \boldsymbol{u}_{\boldsymbol{n} \boldsymbol{b}}^{*}\right)-\Delta t(\nabla p)_{f}=\boldsymbol{u}_{f}^{*}-\Delta t(\nabla p)_{f} .
$$

Note that $L$ is the linear interpolation operator for the cell center $\left(\boldsymbol{u}_{\boldsymbol{P}}\right)$ and the corresponding neighboring velocity $\left(\boldsymbol{u}_{\boldsymbol{n}}\right)$. As the face velocity carries the local pressure gradient, mass flux can be calculated by

$$
F_{f}=\boldsymbol{u}_{f} \cdot \boldsymbol{S}_{f}=\boldsymbol{u}_{f}^{*} \cdot \boldsymbol{S}_{f}-\Delta t(\nabla p)_{f} \cdot \boldsymbol{S}_{f}=F_{f}^{*}-\Delta t(\nabla p)_{f} \cdot \boldsymbol{S}_{f},
$$

where

$$
F_{f}^{*}=u_{f}^{*} \cdot S_{f}
$$

Thus a strong coupling between the ensuing velocity field and the subsequent pressure field is explicitly achieved which prevents spurious oscillations (Verma \& Eswaran 1999) often associated with non-staggered arrangement of variables. A similar time-splitting form in staggered grid was proposed by Kim \& Moin (1985) while Kim \& Choi (2000) treated the pressure with $2^{\text {nd }}$ order accuracy in the unstructured-grid framework. The pressure equation can now be obtained by inserting Eq. (8) into the discrete conservation law, Eq. (3)

$$
\begin{gathered}
\sum_{f} F_{f}^{n+1, l+1}=\sum_{f}\left[F_{f}^{*}-\Delta t(\nabla p)_{f} \cdot S_{f}\right]=0 \\
\Longrightarrow \sum_{f}(\nabla p)_{f} \cdot \boldsymbol{S}_{f}=\frac{1}{\Delta t} \sum_{f} F_{f}^{*} .
\end{gathered}
$$

The pressure field obtained from Eq. (10) projects the velocity field such that the discrete mass conservation law (Eq. 3) is satisfied. As the mass flux can be viewed as the carrier of momentum, it (along with the pressure field) can be used to solve the momentum equations (Eq. 4) which now has become a generic convection-diffusion transport equation owing to its linearity.

\subsection{Implicit treatment of the nonlinearity}

In the present method, the nonlinearity of the governing equations is realized through the prediction of the provisional velocity field seen in Eq. (6). Two different approaches are attempted to resolve it amicably. Though these two alternative routes are formulated keeping in mind a range 
of stiff problems which may arise, they can be used with equal ease. The provisional velocity field, in the absence of a pressure field, is transported by the mass flux leading to the functionality

$$
F^{n+1}=f\left(u_{i}^{*}\right)
$$

3.2a Linearized form $(L M)$ : If the convective flux is linearized to separate out the available mass flux and the provisional velocity field to be solved it takes the form

$$
\left(F_{f} u_{i, f}\right)^{*}=F^{n+1, l} u_{i, f}^{*},
$$

where $l$ denotes the latest available mass flux and should be corrected subsequently based on the converged pressure field using Eq. (8). Thus, Eqs. (6), (8) and (10) have to be iterated until flux-convergence is achieved, i.e.,

$$
F_{f}^{n+1, l} \longrightarrow F_{f}^{n+1}
$$

Essentially this procedure uses flux iteration (denoted here by $l$ ) to resolve the nonlinear convective fluxes. It should be noted here that such linearization is only first-order accurate in progressive estimates, i.e., $O\left(\phi^{n+1, l+1}-\phi^{n+1, l}\right)$. Thus, owing to highly localized acceleration (or deceleration) fluxes may not converge fast rendering the method to be slow. This can be avoided by using a smaller $\Delta t$ which makes the whole time integration technique stable. However, in such a situation purpose of using the implicit method is partially defeated. This issue is likely to occur in problems with strong shear, violent 3D transition or sudden changes in the flow. Though this method consistently treats the governing set of equations implicitly, the pressure equation which is likely to consume majority of the computational resources has to be solved a number of times. The second alternative approach stems from this feature of the linearized form.

3.2b Non-linear form (NM): If the equation depicting the provisional velocity field (Eq. 6) is not linearized, it takes the form

$$
\begin{aligned}
u_{i, P}^{*}+\frac{\Delta t}{V_{P}} \sum_{f}\left[c_{1} F_{f}^{*} u_{i, f}^{*}-\frac{1}{2 R e} F_{d f i}^{*}\right] & =u_{i, P}^{n}-\frac{\Delta t}{V_{P}} \sum_{f}\left[c_{2} F_{f}^{n} u_{i, f}^{n}\right. \\
& \left.+c_{3} F_{f}^{n-1} u_{i, f}^{n-1}-\frac{1}{2 R e} F_{d f i}^{n}\right],
\end{aligned}
$$

which can be cast into a set of nonlinear discrete equations

$$
N_{i}\left(u_{j}^{*}\right)=0
$$

where $i$ and $j$ refer to the equation and variable index. This system can be solved using Newton's method which is computationally cheap, given by the formula

$$
u_{j}^{* k+1}=u_{j}^{* k}-\alpha\left(\frac{\partial N_{p}^{k}}{\partial u_{j}^{*}}\right)^{-1} N_{p}\left(u^{*}\right)^{k}
$$

Though this technique is gifted with fast quadratic convergence, inversion of the Jacobian matrix $\partial N_{i} / \partial u_{j}^{*}$ is not straight forward. Note that the structure of the Jacobian matrix is banded as Eq. (12) contains only immediate (4 in $2 \mathrm{D}$ and 6 in 3D) neighbours. However, if this matrix is 
assumed to be diagonal which is an approximation to the original sparse banded one, the formula simplifies to

$$
u_{j}^{* k+1}=u_{j}^{* k}-\alpha \frac{N_{j}\left(u^{*}\right)^{k}}{a_{P}},
$$

where $a_{P}$ is the diagonal entry for the Jacobian matrix, given by

$$
a_{P}=1+\frac{\Delta t}{V_{P}}\left[\sum_{f}\left(F_{f} \beta_{f}-\frac{1}{2 R e} \gamma_{f}\right)\right],
$$

where $\beta_{f}$ and $\gamma_{f}$ are geometric parameters that appear in the approximation of surface integrals of convective and diffusive fluxes, respectively. This approximation results in cheap and fast convergence of Eq. (12). However, owing to the diagonal approximation this method is expected to fail for finer meshes as the interaction between neighbouring nodes become enhanced. Thus, to compensate for the "poor" initial guess, possible instability and considerable non-uniformity near solid boundaries the updates may need to be under-relaxed.

The above procedure comprising Eqs. (6) (or 12), (9), (10) and (8) lead to a pressure field (and thus the mass flux) that satisfies mass conservation law, but not necessarily a divergencefree velocity field. This can be ensured by solving Eq. (4) which now has become linear with respect to $u_{i}^{n+1}$. Note that the issue of nonlinearity arises only for the implicit $(\mathrm{CN})$, and not for the explicit $(\mathrm{ABCN})$ time marching procedure.

\subsection{Immersed boundary formulation}

The present immersed boundary method is based on direct discrete forcing approach where Cartesian grids are laid on a solid object and the boundary conditions are forced on a number of discrete points $\left(\Omega_{S}\right)$ on the surface of the object. The computational cells are categorized as the fluid $\left(\Omega_{f}\right)$ and solid cells based on the cell centers lying outside and inside the object, respectively, as shown in figure 1 which is a simple illustration. Among the solid cells, those having at least one fluid cell to as its neighbour are termed as the inner cell $\left(\Omega_{I}\right)$ while the neighbouring fluid cells are hereafter referre to as the band cells $\left(\Omega_{B}\right)$. In direct forcing approach the inner cells are treated as field function $(g)$ of band cells that enforces required boundary conditions $\left(\phi_{b}\right)$ on the forcing points.

$$
\phi\left(\Omega_{I}\right)=g\left\{\phi\left(\Omega_{B}\right)\right\} \quad \text { such that } \phi_{s}=\phi_{b} .
$$

Thus, during calculations, the inner cells that appear as neighbouring stencil for band cells, are required to be prescribed so that these band cells "sense" the proximity of the wall.

A blown-up view of a portion of the immersed boundary is shown in figure 2 where $P, N$ and $b$ are typical inner cell, its fluid neighbour (band cell) and the forcing point, respectively, while $Q$ is the mirror point obtained by constructing the surface normal $\boldsymbol{n}$ through $P$. The relevant band points $\left(N_{1}, N_{2} \cdots\right)$ can be extrapolated to calculate the unknown either directly on the forcing points $\left(b_{1}, b_{2} \cdots\right)$ or on the mirror points $\left(Q_{1}, Q_{2} \cdots\right)$. Tseng \& Ferziger (2003) used the second alternative as they found unstable solutions for the cases when the band cells are too close to the surface. However, as this approach uses $\phi\left(P_{1}\right)=2 \phi_{b}-\phi\left(Q_{1}\right)$, the boundary forcing is achieved in a stencil $\left(P_{1} Q_{1}\right)$ twice as large as the original distance $\left(P_{1} b_{1}\right)$. In the present work, two extrapolation procedures are employed with the boundary conditions forced on the surface points. In doing so it has been observed that with progressive refinement if the 


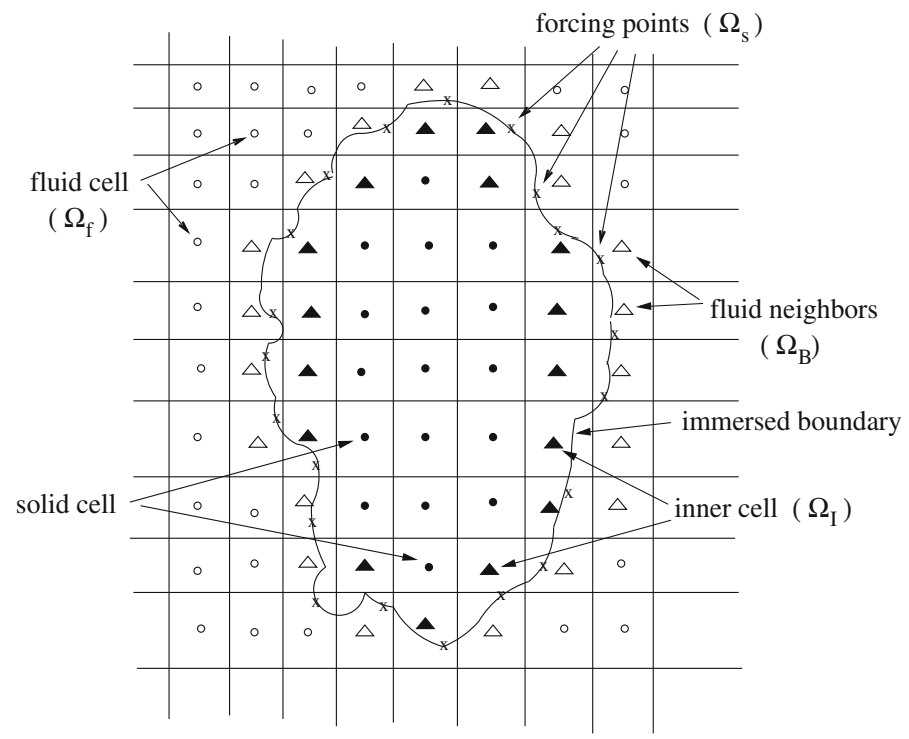

Figure 1. Definition of different category of cells in connection to the immersed boundary.

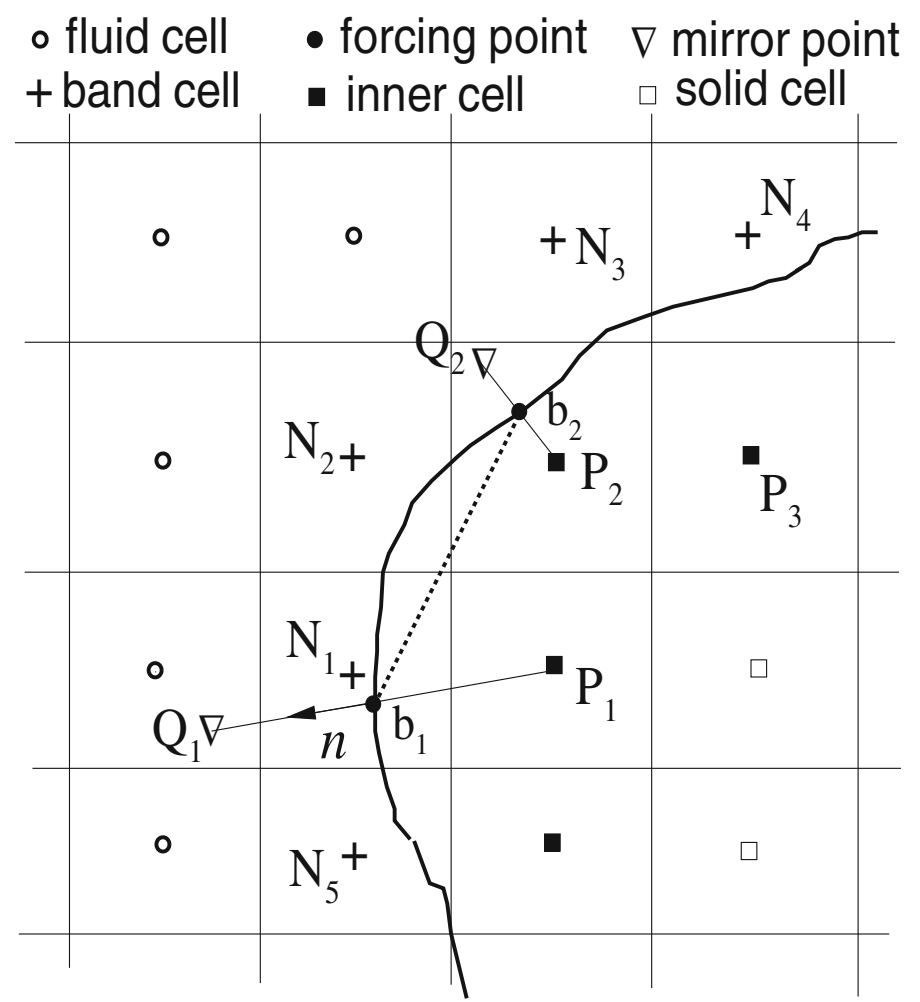

Figure 2. Geometric construction of the immersed boundary. 
inner points that are very close to the surface are modified as the fluid points, the convergence behaviour improves significantly. The criterion that has been consistently used here is an inner point modifies to a fluid point if its distance from the immersed boundary is less than $5 \%$ of the cell representative length, $(\Delta x \Delta y)^{1 / 2}$ or $(\Delta x \Delta y \Delta z)^{1 / 3}$.

Linear/Bi-linear reconstruction: A linear or bi-linear (relevant extra Bi-linear terms underlined) polynomial approximation

$$
\phi=a+b x+c y+\underline{d x y} \text { in } 2 \mathrm{D} \text { and } \phi=a+b x+c y+e z \text { in } 3 \mathrm{D}
$$

can be used to extrapolate $\phi$ at the desired location $\left(\Omega_{I}\right)$. If an inner point has $\phi_{1}, \phi_{2}, \phi_{3}$ and $\phi_{b}$ as its neighbouring band points and the forcing point, respectively, then prescription of Dirichlet condition, $\phi=\phi_{b}$ leads to a linear system $A x=B$ where

$$
A=\left[\begin{array}{cccc}
1 & x_{1} & y_{1} & \frac{x_{1} y_{1}}{\overline{x_{2} y_{2}}} \\
1 & x_{2} & y_{2} & \underline{\underline{x_{3} y_{3}}} \\
\frac{1}{1} & \frac{x_{3}}{x_{b}} & \frac{y_{3}}{y_{b}} & \underline{\underline{x_{b} y_{b}}}
\end{array}\right] \text { and }\left[\begin{array}{cccc}
1 & x_{1} & y_{1} & z_{1} \\
1 & x_{2} & y_{2} & z_{2} \\
1 & x_{3} & y_{3} & z_{3} \\
1 & x_{b} & y_{b} & z_{b}
\end{array}\right]
$$

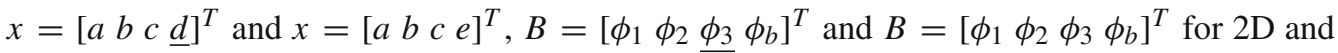
$3 \mathrm{D}$, respectively. The homogeneous Neumann condition on the boundary given by $\partial \phi / \partial n=0$ can be cast into the form

$$
\nabla \phi \cdot \boldsymbol{n}=0
$$

which yields $\left(b+d y_{b}\right) n_{x}+\left(c+\underline{d x_{b}}\right) n_{y}=0$ and $b n_{x}+c n_{y}+e n_{z}=0$ in 2D and 3D, respectively. Thus, $A$ and $B$ of the above linear system change to

$$
\left[\begin{array}{cccc}
1 & x_{1} & y_{1} & \frac{x_{1} y_{1}}{x_{2} y_{2}} \\
1 & x_{2} & y_{2} & \frac{\underline{x_{3} y_{3}}}{1} \\
\frac{1}{0} & \frac{x_{3}}{n_{x}} & \frac{y_{3}}{n_{y}} & \underline{x_{b} n_{y}+y_{b} n_{x}}
\end{array}\right],\left[\begin{array}{cccc}
1 & x_{1} & y_{1} & z_{1} \\
1 & x_{2} & y_{2} & z_{2} \\
1 & x_{3} & y_{3} & z_{3} \\
0 & n_{x} & n_{y} & n_{z}
\end{array}\right]
$$

and $\left[\phi_{1} \phi_{2} \phi_{3} 0\right]^{T},\left[\phi_{1} \phi_{2} \phi_{3} 0\right]^{T}$, respectively.

In the present work, no attempt has been made to use theoretically higher order $\left[>O\left(\Delta x^{2}\right)\right]$ accurate interpolation scheme. The primary reason being it was demonstrated by Majumdar et al (2001) that use of quadratic (or higher order) polynomials does not improve the quality of solution. Moreover, it can be observed that as the choice of neighbouring band cells directly influences forcing of boundary conditions, convergence behaviour is likely to change if the coefficient matrix $A$ becomes near-singular. With grid refinement, the rows of $A$ become similar to each other leading to small determinant, and more so when the number of rows are more than 4. Thus, use of higher order polynomial is not favourable to grid refinement which is detrimental to the quality of solution. As the primary drawback of Cartesian grid methods is its inability to achieve systematic refinement near rigid boundaries, it is better to use overall refined mesh than to use higher-order interpolation polynomial for the forcing of boundary conditions. 


\subsection{Linear solver}

All set of linear equations arising during the time marching procedure can be cast into a form

$$
A_{f} \phi=b_{f}
$$

where the linear operator $A_{f}$ corresponds to a specific stencil size decided by the convective scheme (and less likely by the diffusion term) used. However, the grids being Cartesian, the pressure equation (Eq. 10) results in a simple penta-diagonal (2D) or septa-diagonal (3D) matrix structure. The reconstruction of a variable near the immersed boundary yields a linear relation among an inner cell and a number of band cells.

$$
A_{b} \phi=b_{b}
$$

Note that the above system is same as the one described in section 3.3. Equation sets (21) and (22) need to be solved simultaneously so that progressive changes in the inner cell values force the boundary conditions on the desired locations on the immersed boundary.

In the present work, the stabilized version of the Bi-Conjugate Gradient method (Zhang 1997) has been used for the velocity steps (Eqs. 4 and 6) while Stone's Strongly Implicit Procedure (SIP) (Stone 1968) has been used as a pre-conditioner (Ferziger \& Perić 1996) for monotonic and rapid convergence of the pressure Poisson equation (Eq. 10). A brief description of the SIP factorization along with the diagonals is provided in Appendix A.

\subsection{Parallelization strategy}

The three-dimensional numerical code has been parallelized based on distributed memory allocation. The computational domain is decomposed into a number of sub-domains and each of them is assigned to a computing processor. At the interfaces of these sub-domains, communication of solution variables are performed in the MPI environment to synchronize computations among the processors. Though computational load on each processor should be theoretically same so as to achieve optimum performance, it is often compromised because of grid distribution and nature of the solution. If a set of inner cells and the corresponding band cells lie in a single processor, extra communications do not arise. However, when they are distributed among

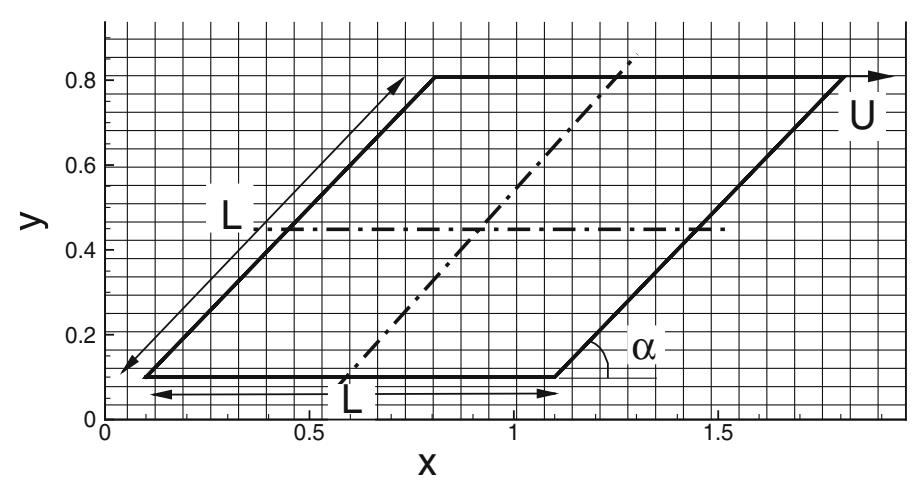

Figure 3. Geometric detail and the Cartesian mesh (every fourth grid line is shown) used for the inclined cavity problem. 

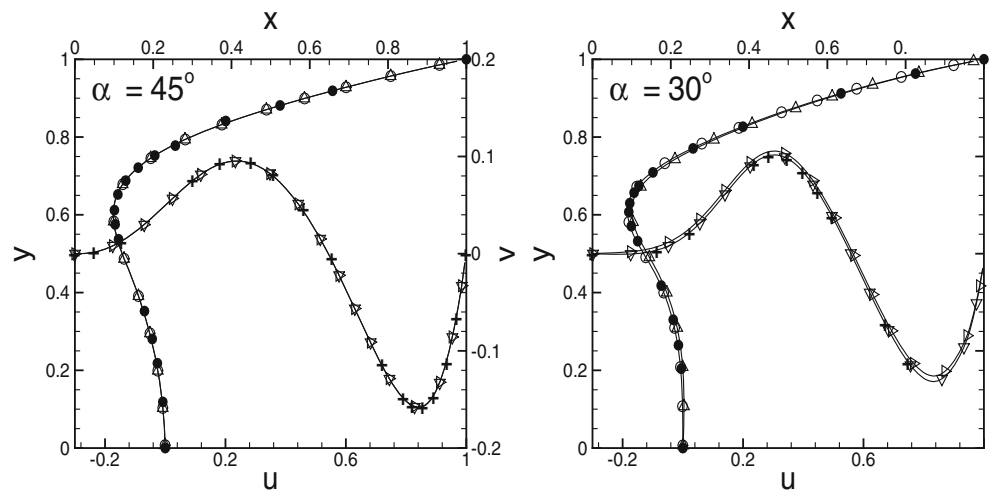

Figure 4. Mid-sectional velocities for the vortical flow in the inclined cavity. $-0-: u$, linear; $-\Delta-: u$, bilinear; $-\nabla-: v$, linear; $\longrightarrow \triangleright-: v$, bi-linear correspond to present data while $\bullet: u$ and $+: v$ refer to Demirdžić et al (1992).

different processors either the original sub-domains has to be restructured at the cost of computational imbalance or complex communication paths have to be determined. The present method has resorted to the first alternative so that extra communication is avoided. Preliminary tests have shown that the overall parallel code scales well with processors given the problem size is large enough. Though BiCGSTAB algorithm can be parallelized it is the ILU-type of factorization and its subsequent forward and backward sweeps, that accelerates the convergence, can only be made partially parallel owing to its sequential data dependence.

\section{Results}

The present immersed boundary method has been applied to flows in reasonably complex geometries covering both homogeneous and non-homogeneous Dirichlet boundary conditions for the velocities. However, as the method does not intend to venture into the high Reynolds number regime, the problems are so chosen that its capability to handle laminar unsteady flows or 2D-to-3D transitional flows are appropriately judged.

\subsection{Vortical flow in an inclined cavity}

Steady vortical flow in an inclined cavity whose geometric details are shown in figure 3 , is a suitable test of boundary non-orthogonality. Simulations have been carried out for $\alpha=30^{\circ}$ and
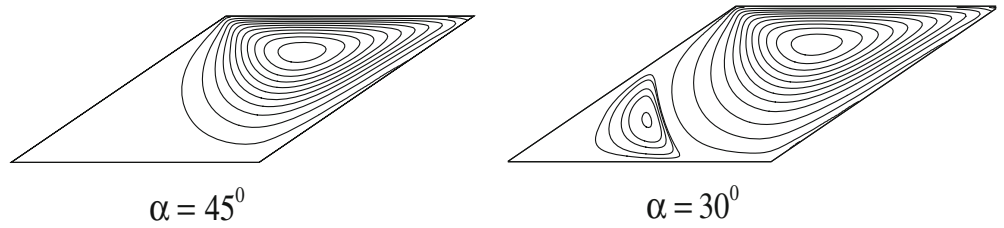

Figure 5. Computed stream lines inside the inclined cavity. 


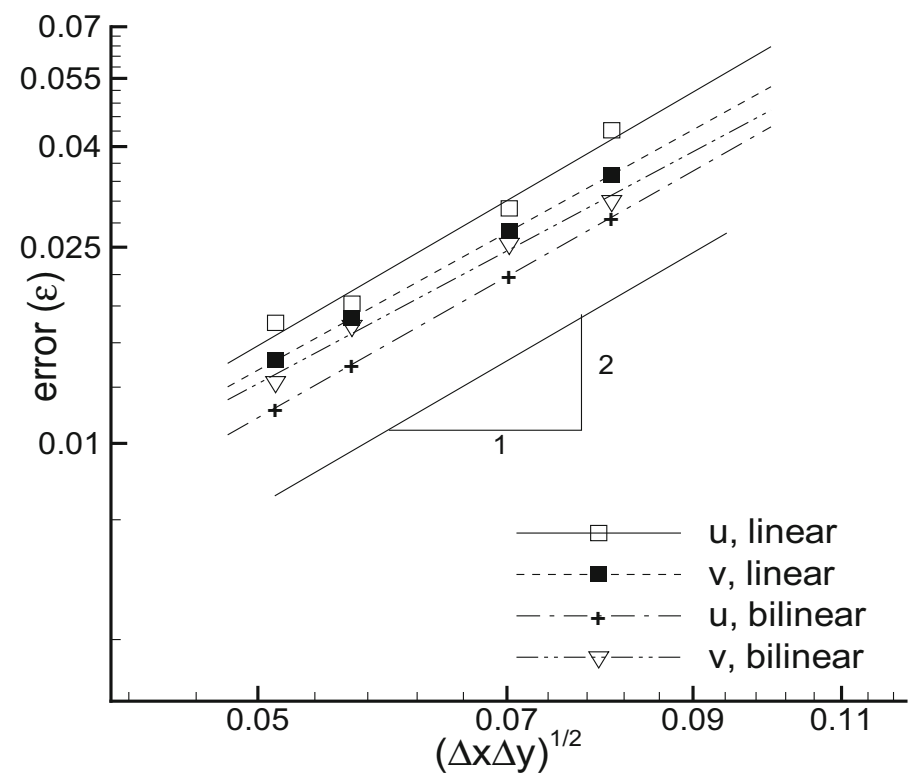

Figure 6. Test of spatial accuracy for flow past a circular cylinder.

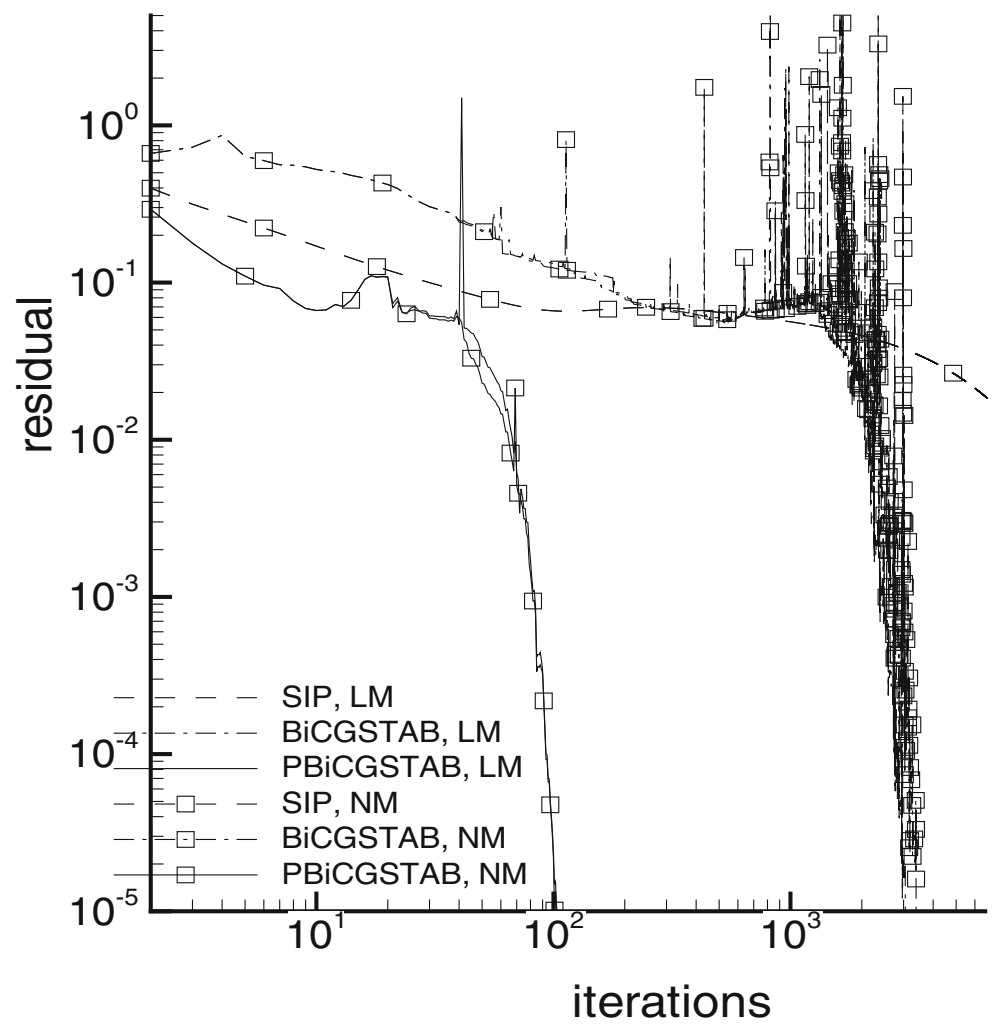

Figure 7. Behaviour of the linear solvers applied with the two linearization forms. 

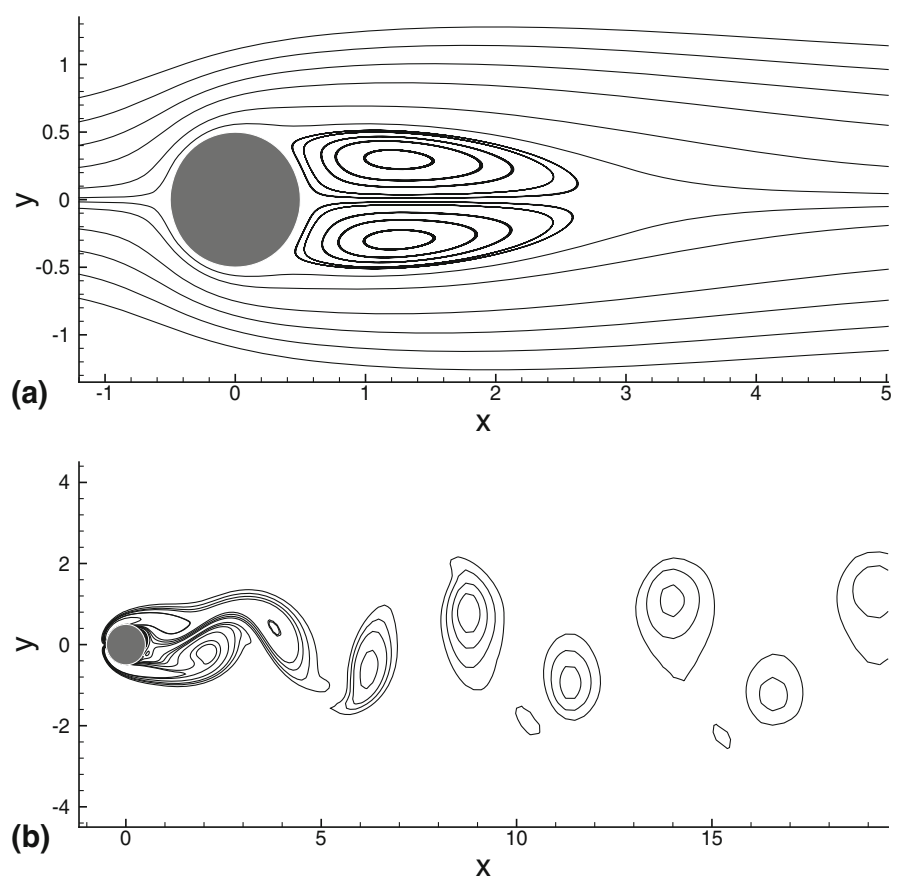

Figure 8. Flow pattern behind the circular cylinder; (a) stream lines at $R e=40$ and (b) instantaneous vorticity contours (negative $\omega_{z}$ in dashed line) at $R e=100$.

$45^{\circ}$ at $\operatorname{Re}(U L / v)=100$ with a uniform mesh of $160 \times 80$. The steady velocity profile along mid-width and mid-height, shown in figure 4, compare well with Demirdžić et al (1992) where computations were performed on a body fitted refined mesh. It can be seen that the improvement in solution accuracy by using bi-linear extrapolation as the reconstruction scheme is marginal. This observation is consistent with earlier studies on discrete forcing approach. In view of possible singularities (in the coefficient matrix of Eqs. (18) and (20) arising from specific grid arrangement and marginal improvement in the solution accuracy it is advisable to use linear extrapolation as the reconstruction scheme. The computed streamlines are shown in figure 5 where the secondary vortex at $\alpha=30^{\circ}$ is correctly resolved.

Table 1. Comparison of force coefficients obtained from the two non-linear formulations with previous studies for flow past a circular cylinder.

\begin{tabular}{|c|c|c|c|c|}
\hline & \multirow{2}{*}{$\frac{R e=40}{C_{d}}$} & \multicolumn{3}{|c|}{$\operatorname{Re}=100$} \\
\hline & & $S t$ & $<C_{d}>$ & $C_{L}^{\prime}$ \\
\hline Tseng \& Ferziger (2003) & 1.53 & 0.164 & 1.42 & 0.29 \\
\hline Kim et al (2001) & 1.51 & 0.165 & 1.33 & - \\
\hline Present, LM,linear & 1.57 & 0.171 & 1.455 & 0.311 \\
\hline Present, NM,linear & 1.59 & 0.169 & 1.47 & 0.298 \\
\hline Present, LM,bi-linear & 1.53 & 0.168 & 1.462 & 0.3 \\
\hline Present, NM,bi-linear & 1.56 & 0.17 & 1.448 & 0.317 \\
\hline
\end{tabular}




$$
q=\pi / 2
$$
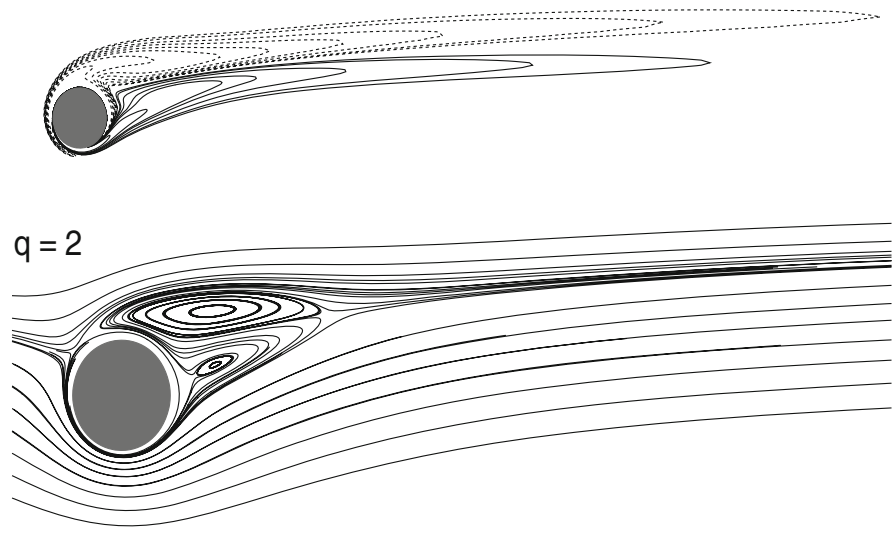

Figure 9. Instantaneous vorticity field at $q=\pi / 2$ and steady stream lines at $q=2$ for flow past a rotating cylinder.

\subsection{Flow past a stationary/rotating circular cylinder}

Flow past a circular cylinder is an excellent choice for simulation in complex geometries as it offers a rich combination of geometric and physical complexities. In the present study, both the stationary and rotating cases are examined with the proposed technique. The physical problem involves unbounded flow $\left(U_{\infty}\right)$ past a circular cylinder of diameter $d$ placed at the origin. The

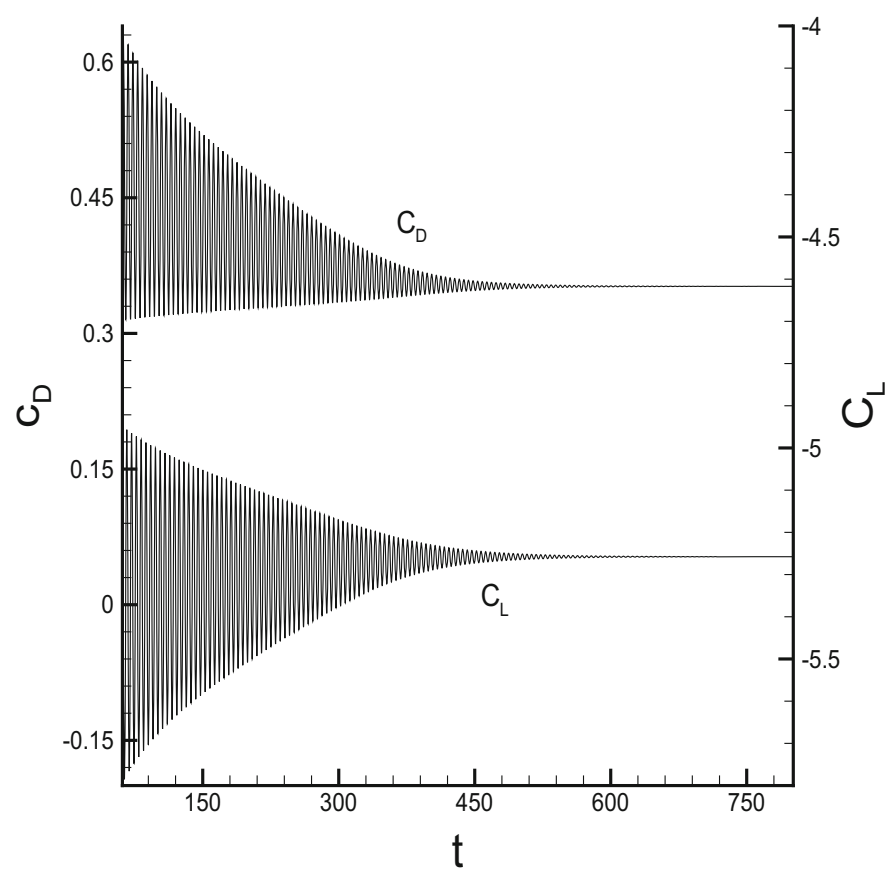

Figure 10. Average drag and lift coefficients showing attainment of a asymptotic steady state at $q=2$. 
Table 2. Force coefficients for flow past a rotating circular cylinder.

\begin{tabular}{lcccccc}
\hline & \multicolumn{3}{c}{$q=\pi / 2$} & & \multicolumn{2}{c}{$q=2$} \\
\cline { 2 - 4 } \cline { 6 - 7 } & $\left\langle C_{d}\right\rangle$ & $\left.<C_{L}\right\rangle$ & $S t$ & & $C_{d}$ & $C_{L}$ \\
\hline Maruoka (2003) & 0.84 & -4.11 & 0.191 & & - & - \\
Mittal \& Kumar (2003) & - & - & - & & 0.31 & -5.29 \\
Zhang et al (2008) & 0.77 & -3.98 & 0.191 & & 0.39 & -5.26 \\
Present & 0.90 & -4.109 & 0.193 & & 0.352 & -5.258 \\
\hline
\end{tabular}

upstream, downstream and the cross-stream extent of the computational domain are chosen as $5 d, 25 d$ and $16 d$, respectively, and Reynolds number is defined as $R e=U_{\infty} d / \nu$. The nonuniform Cartesian grid used for the calculations is $250 \times 140$ with a refined square block of size $1.1 d$ consisting of uniform grid $(60 \times 60)$ is wrapped around the cylinder to resolve the flow near the cylinder and in the near wake. For the rotating (counterclockwise with angular speed $\omega$ ) cylinder case, the tip velocity is defined as $q=d \omega / 2 U_{\infty}$. In all the subsequent test problems the Orlanski boundary condition, given by $\partial \phi / \partial t+U_{a v} \partial \phi / \partial n=0$ has been used where $\phi, U_{a v}, n$ refer to velocity components, average stream-wise velocity at the exit and stream-wise direction, respectively. This condition being similar to the first-order linear wave equation transports ensuing flow structure without any distortion.
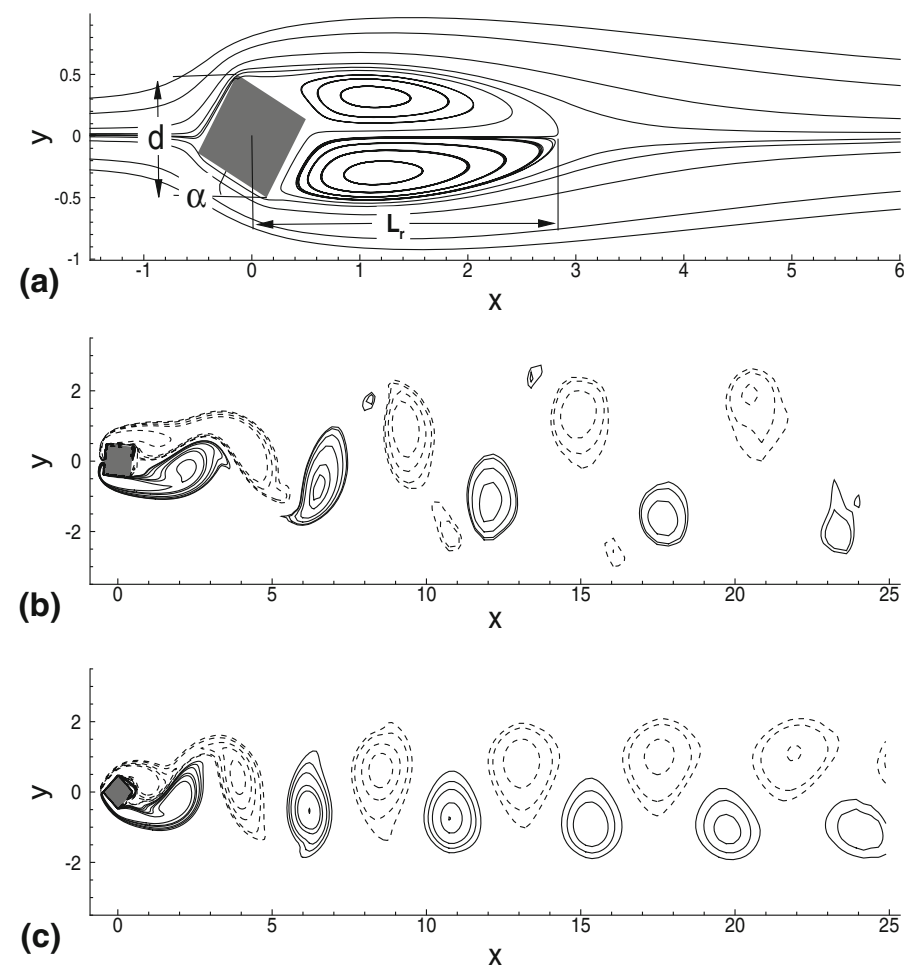

Figure 11. Wake behind the inclined cylinder; (a) steady separation bubble at $R e=40, \alpha=22.7^{\circ}$, instantaneous vorticity field (negative $\omega_{z}$ in dashed line) at $R e=100$ for (b) $\alpha=6^{\circ}$ and (c) $\alpha=45^{\circ}$. 
To test the actual order of accuracy of the numerical solution obtained from the two reconstruction schemes, a domain $(-2 d \leq x, y \leq 2 d)$ including the cylinder is chosen. Computations have been performed on progressively coarser mesh with results of the finest mesh $(250 \times 140)$ taken as the benchmark solution. The solution at a coarser mesh is interpolated to the finer mesh for comparison and is shown in figure 6 . The error $(\epsilon)$ in the figure is defined by the $l_{2}$ norm of the difference between the benchmark and the interpolated solutions. Both the extrapolation procedures yield a convergence behaviour $\epsilon \propto(\Delta x \Delta y)^{n / 2}$ with $n \approx 1.9$. The loss in accuracy can be attributed to the combined effects of overall solution error and the extrapolation error at the boundary. No appreciable difference among the two implicit formulations (LM and NM) is observed. Figure 7 shows the relative convergence behaviour of the different linear solvers when applied with the two implicit formulations. Rate of convergence improves significantly from the SIP method to the PBiCGSTAB technique while BiCGSTAB lies between them with nonmonotonic convergence. However, it is observed that with the non-linear form, PBiCGSTAB gives a smooth convergence excluding the possibility of occasional instabilities of the linear form (LM). The steady state streamlines and instantaneous vorticity contours at $R e=40$ and 100 are shown in figure 8 . The average force coefficients and strouhal number is compared in
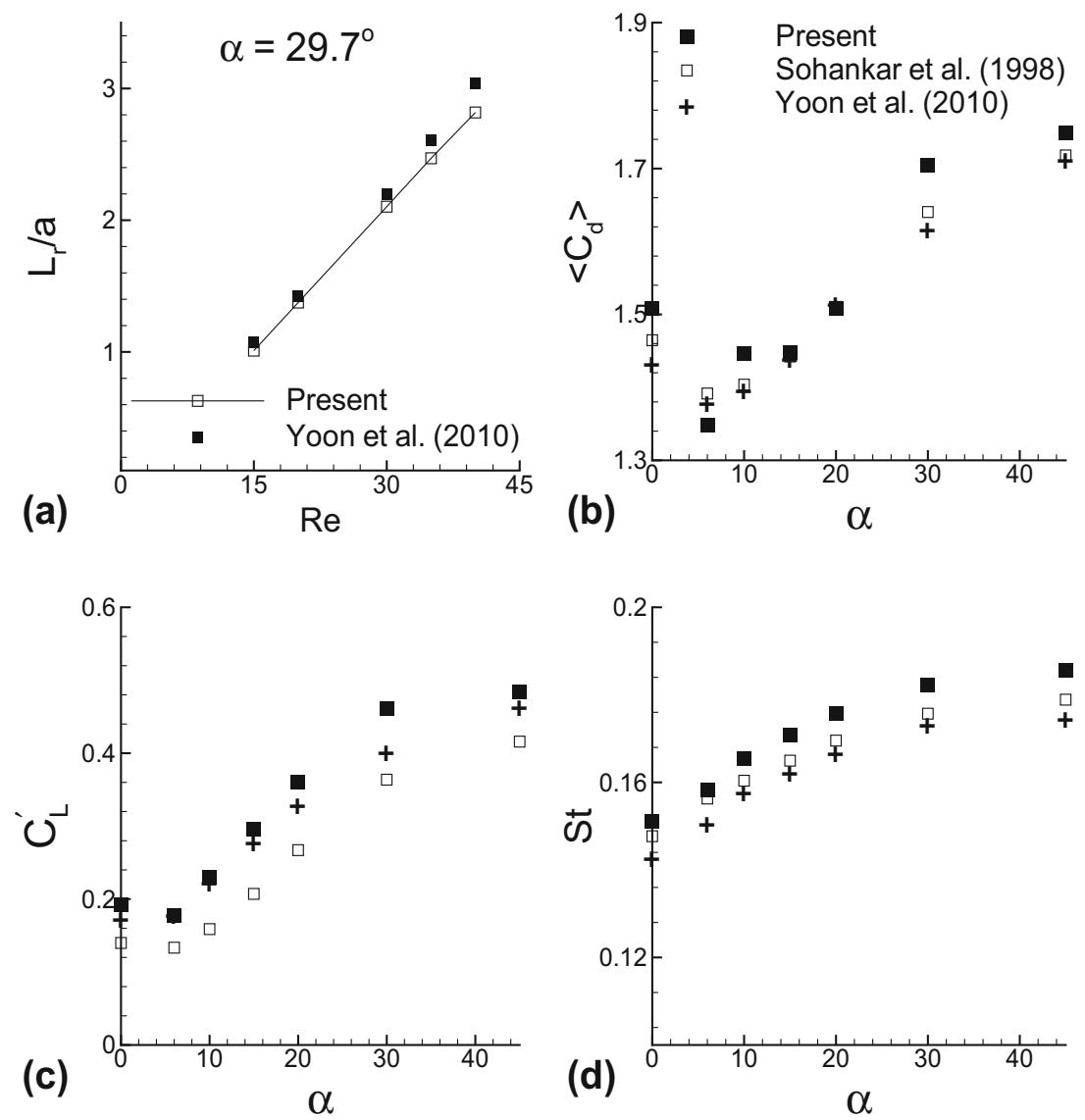

Figure 12. Comparison of (a) re-attachment length at $\alpha=29.7^{\circ}$, (b) average drag coefficient, (c) root mean square of the lift coefficient and (d) Strouhal number at $R e=100$. 
Table 3. Computational details for three-dimensional flow past a circular cylinder.

\begin{tabular}{llcl}
\hline$R e$ & mesh & band cells & $\Delta t$ \\
\hline 200 & $225 \times 110 \times 80$ & 6320 & 0.01 \\
300 & $241 \times 120 \times 100$ & 9108 & 0.005 \\
\hline
\end{tabular}

table 1 which reemphasizes the marginal improvement of solution accuracy due to the bi-linear extrapolation scheme and near- identical prediction by the two implicit formulations.

The rotating cylinder case tests the ability of the method for non-homogeneous boundary conditions as the boundary velocity is the tangential velocity of the cylinder that changes in the azimuthal direction. All the computational features are kept same as of the stationary case and simulations have been carried out for $q=\pi / 2$ and 2 at $R e=200$. In agreement to Zhang et al (2008), the flow is found to be time-periodic at $q=\pi / 2$ while long time simulation shows that the wake oscillation decays slowly to reach the steady state at $q=2$. Instantaneous vorticity contours and steady state streamlines are shown in figure 9 while attainment of the asymptotic

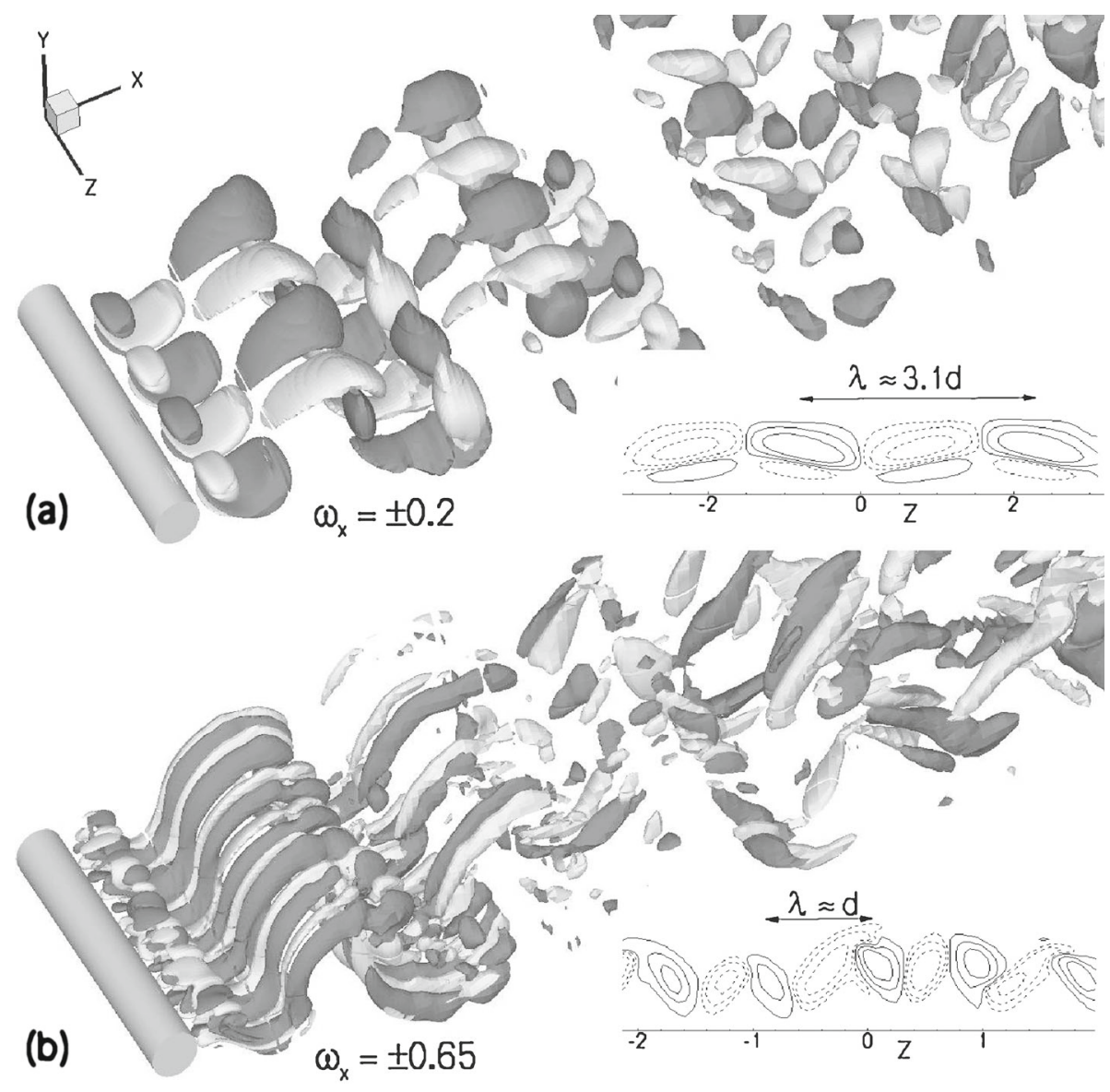

Figure 13. Span-wise vorticity at (a) $R e=200$ and (b) $R e=300$ behind the circular cylinder; Mode A and $\mathrm{B}$ instabilities are shown with the span-wise wavelength. 
steady state can be seen form the drag and lift coefficient signal, shown in figure 10 . The average force coefficients, shown in table 2 , are close to the reported values.

\subsection{Flow past a inclined cylinder}

As an example of flow over a body with sharp corners, two-dimensional unbounded flow past a square cylinder of side $a$ placed at an angle $\alpha$ (see figure 11 (a)) with the incoming stream has been tested. The cylinder is located at the origin making a blockage of width $d=a(\cos \alpha+$ $\sin \alpha$ ). Only the nonlinear formulation (NM) is used while all the geometric parameters are kept same as the circular cylinder case. A non-uniform mesh of size $300 \times 150$ is used which has a refined $(60 \times 60)$ block $(|x, y|<0.6 d)$ containing the cylinder with $\Delta t=0.01 U / d$ being used for the time marching.

The steady separation bubble at $\alpha=22.7^{\circ}, R e=40$ is shown in figure 11(a) while vorticity field of two selective cases from the unsteady periodic regime are shown in figures 11(b) and (c) which correspond to $\alpha=6^{\circ}, 45^{\circ}$ and $R e=100$, respectively. The recirculation length $\left(L_{r}\right)$,
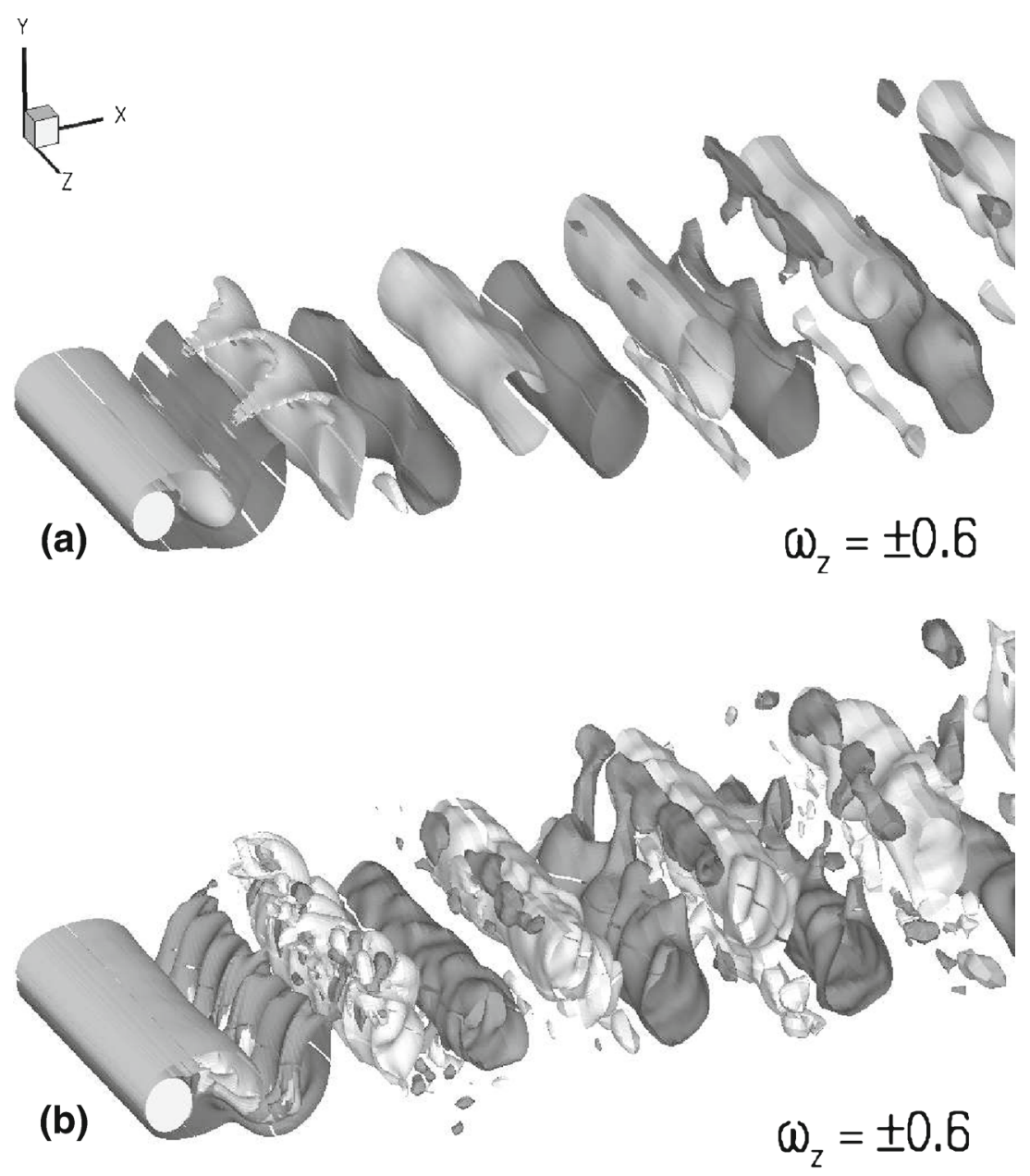

Figure 14. Stream-wise vorticity behind the circular cylinder; (a) $R e=200$ and (b) $R e=300$. 
Table 4. Summary of 2D-3D transitional flow behind a circular cylinder.

\begin{tabular}{lccccccc}
\hline & \multicolumn{3}{c}{$R e=200$} & & \multicolumn{3}{c}{$R e=300$} \\
\cline { 2 - 4 } \cline { 6 - 8 } & $\left.<C_{d}\right\rangle$ & $S t$ & $\lambda_{z} / d$ & & $\left.<C_{d}\right\rangle$ & $S t$ & $\lambda_{z} / d$ \\
\hline Labbé \& Wilson (2003) & 1.318 & 0.195 & $\approx 4$ & & 1.287 & 0.205 & $\approx 1$ \\
Williamson (1988) & - & 0.196 & & - & 0.204 & - \\
Williamson (1996) & - & - & $\approx 3.55$ & & - & - & $\approx 1.05$ \\
Balaras (2004) & - & - & - & & 1.27 & 0.21 & - \\
Present & 1.42 & 0.198 & $\approx 3.1$ & & 1.283 & 0.211 & $\approx 1$ \\
\hline
\end{tabular}

defined as the distance of the saddle point from the cylinder center (shown in figure 11(a)), as a function of $R e$ is shown for $\alpha=22.7^{\circ}$ in figure 12(a). Note that $L_{r}$ is only shown for the steady cases. The average drag coefficient $\left(<C_{d}>\right.$ ), root mean square of the lift coefficient $\left(C_{L}^{\prime}\right)$ and the vortex shedding frequency $(S t)$ are compared in figures 12(b)-(d) with Sohankar et al (1998) and Yoon et al (2010) for the range $0<\alpha<45^{\circ}$ at $R e=100$. The results agree well with two previous works and the maximum difference is found at $\alpha \approx 30^{\circ}$ when the wake shows maximum fluctuation evident from $C_{L}{ }^{\prime}$. However, the difference is acceptable considering the difference in resolution and interval of time integration among the present and the two previous studies.

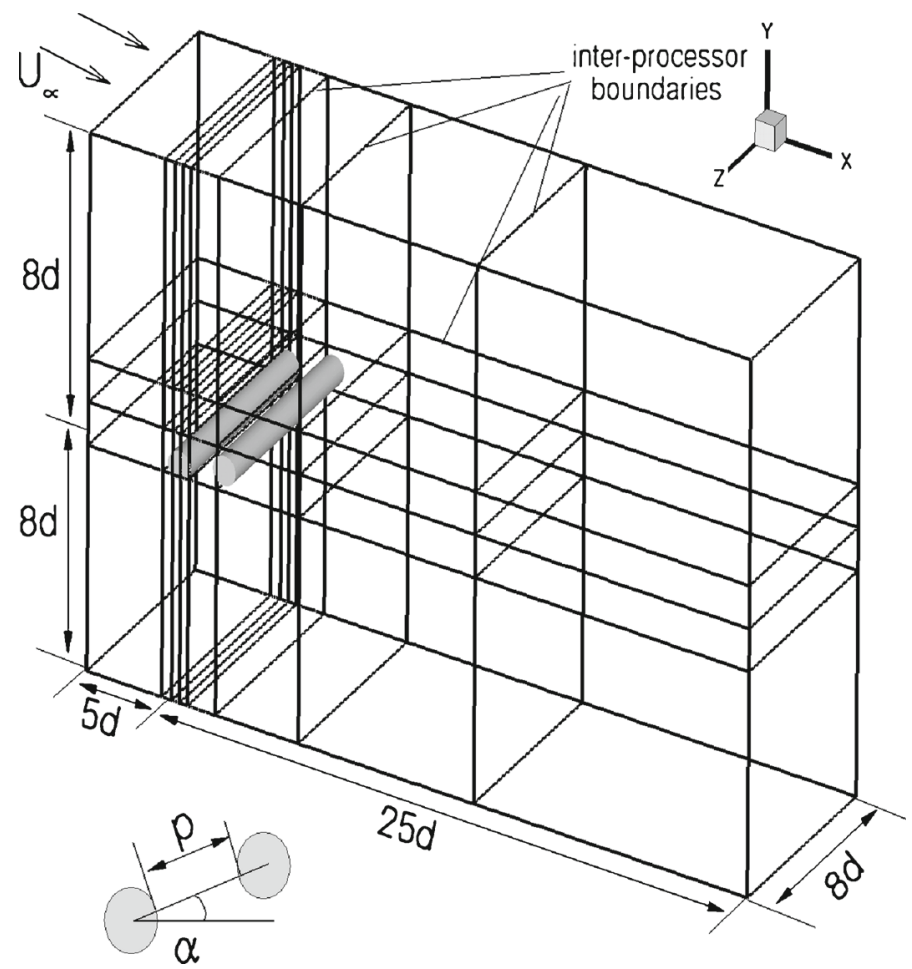

Figure 15. Geometric details for the flow past two staggered cylinders. 


\subsection{Three-dimensional wake transition behind a circular cylinder}

The present technique in its parallelized form has been employed to predict three-dimensional wake transition behind a circular cylinder placed in uniform flow. The span-wise length of the cylinder is taken as $2 \pi d$ while other details are shown in table 3 . The non-uniform mesh described in section 3.3 is uniformly expanded in the span-wise direction. A coarse mesh simulation is first conducted and the dynamically stationary result is interpolated to the chosen grid $(221 \times 151 \times 100)$ which serves as the initial condition for the results shown here. The parallelized code is run on 8 processors for nearly fifty vortex shedding cycles. It has been observed that the pre-conditioned-BiCGSTAB solver takes only a few iterations $(<10)$ to reduce the errors of four orders if the interpolated result from a coarser grid is used as the initial condition.
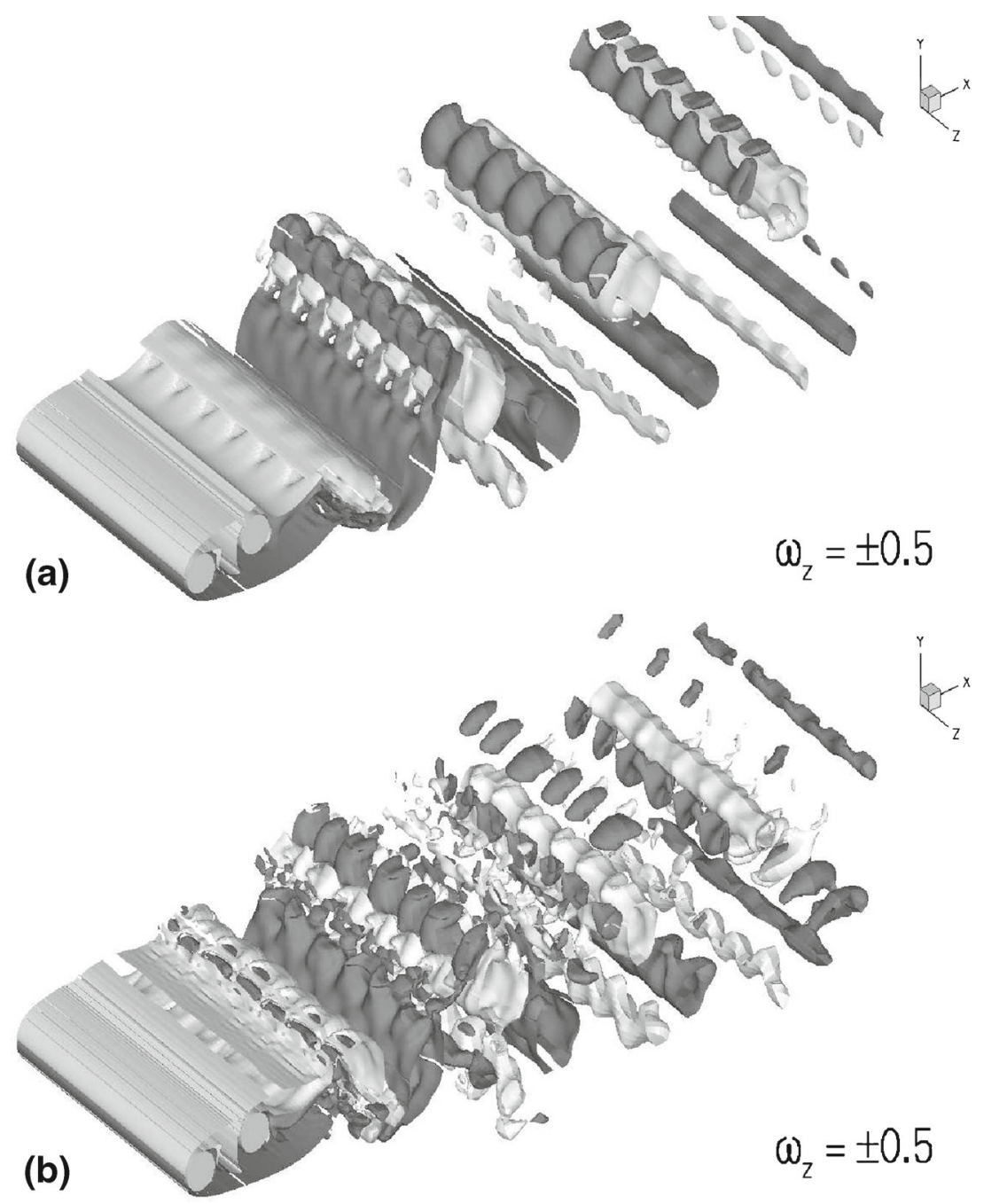

Figure 16. Stream-wise vorticity showing onset of three-dimensionality; (a) $R e=200$ and (b) $R e=$ 300 . 
In the dynamically stationary state, the numerical code takes about 40 minutes to advance one vortex shedding cycle.

It has been observed that the two-dimensional wake shows signs of three-dimensionality at $R e \approx 190$. Around this Reynolds number a three-dimensional instability, known as the mode A instability, occurs whose span-wise wavelength $\left(\lambda_{z} / d\right)$ varies between $3 d$ and $4 d$. At even higher Reynolds number the transition of the vortex shedding mode becomes more complicated and at $R e \approx 300$ the wake exhibits a span-wise wavelength $\lambda_{z} / d \approx 1$. This instability is known as the mode B instability. While in mode A the span-wise vorticity appears in staggered arrangement in the stream-wise direction, they rearrange to in-line configuration in mode B. Figures 13 and 14 show span-wise $\left(\omega_{x}\right)$ and stream-wise $\left(\omega_{z}\right)$ vorticity surfaces at $\operatorname{Re}=200$ (a) and 300 (b). The onset of the instabilities at $R e=200$ and breaking of the von Karman vortex street at $R e=300$ is evident form figures 14 (a) and (b), respectively. The staggered arrangement of the span-wise

(a)
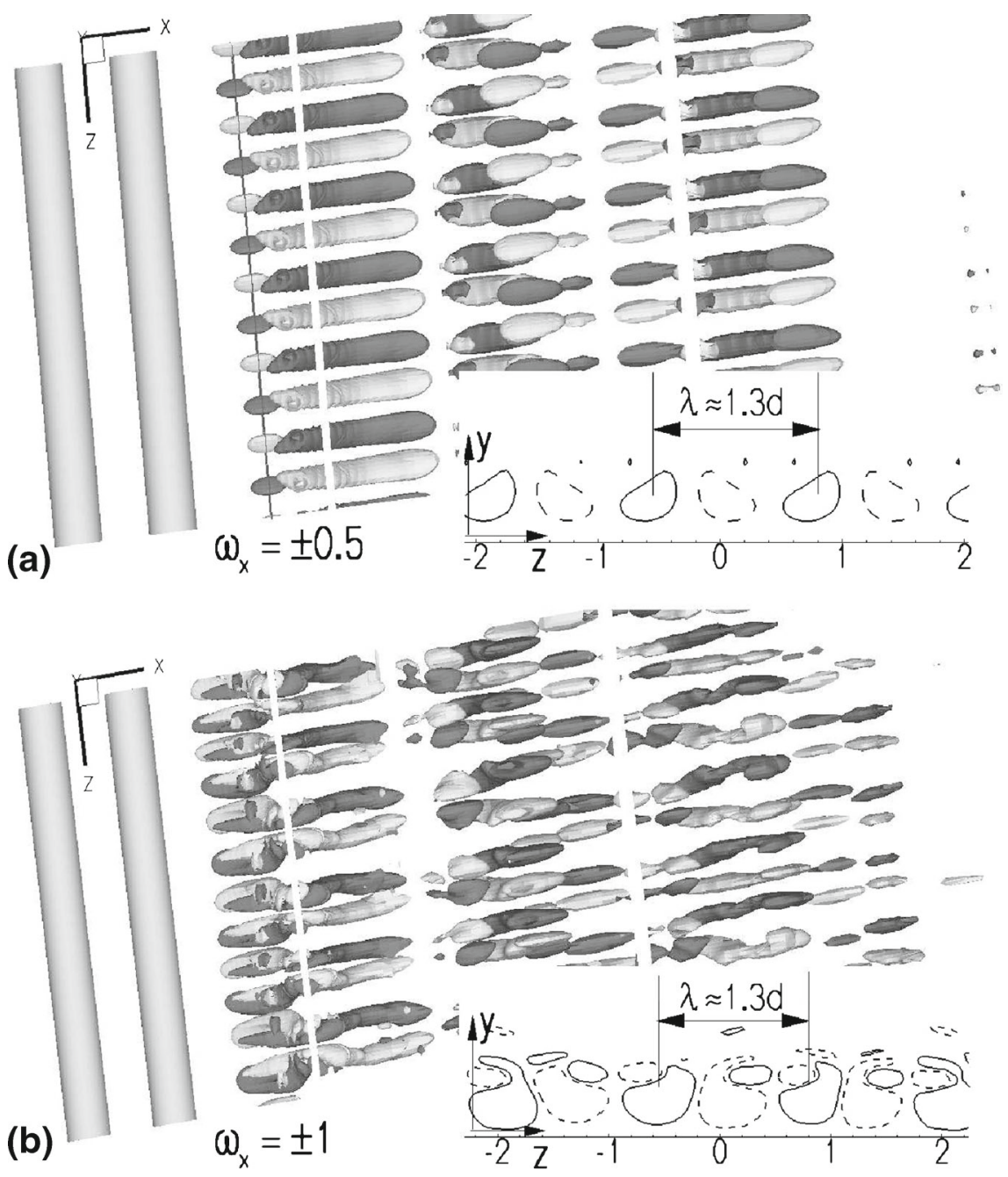

Figure 17. Span-wise vorticity showing the mode of instability; (a) $R e=200$ and (b) $R e=300$. 
vorticity (of mode A) at $R e=200$ is seen to change to in-line pattern (of mode $\mathrm{B}$ ) at $R e=200$ in figures 13 (a) and (b). A detailed view of the span-wise vorticity in the near wake $(x=2$ plane) is shown in the inset of figure 13 which confirms the span-wise wavelength $\left(\lambda_{z} / d\right)$ of the flow instabilities. The average drag coefficient $\left.\left(<C_{d}\right\rangle\right)$, the strouhal frequency $(S t)$ and $\lambda_{z}$ is compared with a few previous studies in table 4.

\subsection{Three-dimensional wake behind two staggered circular cylinders}

The final test case is the flow past two cylinders in staggered arrangement with a pitch ratio $p / d=1$ and angle $\alpha=10^{\circ}$. The geometric details and the decomposition are shown in figure 15. The Reynolds number is defined as $R e=U_{\infty} d / v$ where $U_{\infty}$ and $d$ are the incoming flow velocity and diameter of the cylinders, respectively. Simulations have been performed for $R e=200$ and 300 with a non-uniform mesh $257 \times 202 \times 120$ distributed among 32 computing processors. Three-dimensional wake transition is clearly observed in figure 16 where periodic nature of the stream-wise vorticity at $R e=200$ breaks into irregular pattern at $R e=300$. At this range of Reynolds number, the wake shows sign of mode B instability (see figure 17) as in both the cases span-wise vorticity exhibits a wavelength $\lambda \approx 1.3 d$. Though a regular staggered arrangement of the span-wise vorticity is seen at $R e=200$, complex chain like structure is seen at $R e=300$.

\section{Conclusions}

The objective of the present work was to develop a robust implicit Cartesian grid method based on the discrete forcing approach. The arrangement of variables was chosen in non-staggered form which, in contrast to the staggered form, is known to produce pressure wiggles owing to the lack of velocity-pressure coupling. This issue has been addressed adequately as to how the coupling can be retained. The solution is achieved in predictor-corrector steps where a provisional velocity is predicted without considering the pressure and a localized pressure gradient is applied with the velocity in the corrector step. The non linearity arising due to the implicit treatment of the convective terms has been handled both by linearization as well as a complete non linear system. Both linear and bi-linear extrapolation techniques have been tested for the reconstruction of velocity and pressure on the immersed boundary. The Poisson equation for the pressure has been solved using the pre-conditioned BiCGSTAB method whose convergence behaviour is studied for different reconstruction schemes.

In agreement with the previous studies, marginal improvement in the quality of solution is found by using bi-linear extrapolation while often convergence behaviour deteriorates owing to the ensuing matrix structure. Thus, for three-dimensional computations, linear reconstruction scheme is found to be the optimum choice. An accelerated monotonic convergence behaviour is found when the BiCGSTAB technique is pre-conditioned by the SIP method. However, the solver performance may vary for problems with highly curved boundaries as local refinement is not straight forward for the present method. The two-dimensional test cases with both stationary and moving boundaries show reasonable agreement with the previous experimental studies and body-fitted numerical simulations. In three-dimensional simulations, parallelized calculations yielded expected speed-up with the solution agreeing well with the literature. 


\section{Acknowledgements}

The present research was carried out through the funds available from the institute start-up grant R\&D/07/SG/ME/P/ARKD/1/2009-2010. The author thanks Prof. Vinayak Eswaran for his invaluable inputs.

\section{Appendix A}

The discrete linear equations $(A \phi=b)$ arising from the governing equations can be cast into a septa-diagonal form

$$
a_{B} \phi_{B}+a_{S} \phi_{S}+a_{W} \phi_{W}+a_{P} \phi_{P}+a_{E} \phi_{E}+a_{N} \phi_{N}+a_{T} \phi_{T}=b,
$$

where $a_{B}=a_{T}=0$ in the two-dimensional form. Diagonals of the lower ( $\left.L W, L S, L P, L B\right)$ and upper $(U E, U N, U T)$ triangular factors, obtained by the normal $L U$ factorization procedure along with the implicit relations proposed by Stone (1968), are given below

$$
\begin{aligned}
L S_{P}= & a_{S} /\left(1+\gamma\left(U T_{S}+U E_{S}\right)\right) \\
L B_{P}= & a_{B} /\left(1+\gamma\left(U E_{B}+U N_{B}\right)\right) \\
L W_{P}= & a_{W} /\left(1+\gamma\left(U N_{W}+U T_{W}\right)\right) \\
L P_{P}= & a_{P}+\gamma\left(L W_{P} U N_{W}+L W_{P} U T_{W}+L S_{P} U T_{S}+L S_{P} U E_{S}\right. \\
& \left.+L B_{P} U E_{B}+L B_{P} U N_{B}\right)-L W_{P} U E_{W}-L S_{P} U N_{S}-L B U T_{B} \\
U E_{P}= & \left(a_{E}-\gamma\left(L S_{P} U E_{S}+L B_{P} U E_{B}\right) / L P_{P}\right. \\
U T_{P}= & \left(a_{T}-\gamma\left(L S_{P} U T_{S}+L W_{P} U T_{W}\right)\right) / L P_{P} \\
U N_{P}= & \left(a_{N}-\gamma\left(L W_{P} U N_{W}+L B_{P} U N_{B}\right)\right) L P_{P}
\end{aligned}
$$

with $\gamma$ being a implicit factor and $\gamma \approx 0.9$ gives the best convergence for a range of problems. The above factors reduce the original linear system into a two-step substitution procedure

$$
L U \phi=b \Longrightarrow L M=b \text { and } U \phi=M \text {, }
$$

\section{References}

Angot P, Bruneau C H and Frabrie P 1999 A penalization method to take into account obstacles in viscous flows. Numer. Math. 81: 497-520

Armfield S W 1991 Finite difference solutions of the Navier-Stokes equations on staggered and nonstaggered grids. Comput. Fluids 20: 1-17

Arthurs K M, Moore L C, Peskin C S, Pitman E B and Layton H E 1998 Modeling arteriolar flow and mass transport using the immersed boundary method. J. Comput. Phys. 147: 402-440

Balaras E 2004 Modeling complex boundaries using an external force field on fixed Cartesian grids in large-eddy simulations. Comput. Fluids 33(3): 375-404

Barton I E and Kirby R 2000 Finite difference scheme for the solution of fluid flow problems on nonstaggered grids. Int. J. Numer. Methods Fluids 33: 939-959

Berthelsen P A and Ytrehus T 2007 Stratified smooth two-phase flow using the immersed interface method. Comput. Fluids 36(7): 1273-1289

Borazjani I, Ge L and Sotiropoulos F 2008 Curvilinear immersed boundary method for simulating fluid structure interaction with complex 3d rigid bodies. J. Comput. Phys. 227(16): 7587-7620 
Bottino D C 1998 Modeling viscoelastic networks and cell deformation in the context of the immersed boundary method. J. Comput. Phys. 147: 86

Demirdžić I, Lilek Ž and Perić M 1992 Fluid flow and heat transfer test problems for non-orthogonal grids: benchmark solutions. Int. J. Numer. Meth. Fluids 15: 329-354

Denaro F M and Sarghini F 2002 2-d transmitral flows simulation by means of the immersed boundary method on unstructured grids. Int. J. Numer. Methods Fluids 38: 1133-1158

der Wijngaart R J F V 1990 Composite grid techniques and adaptive mesh refinement in computational fluid dynamics. Report CLaSSiC-90-97

Fadlun E A, Verzicco R, Orlandi P and Mohd-Yusof J 2000 Combined immersed-boundary finite-difference methods for three-dimensional complex flow simulations. J. Comput. Phys. 161(1): 35-60

Fauci L J 1990 Interaction of oscillating filamentsa computational study. J. Comput. Phys. 86: 294

Ferziger J H and Perić M 1996 Computational methods for fluid dynamics. Springer 95-106

Fogelson A L and Peskin C S 1988 A fast numerical method for solving three-dimensional stokes equations in the presence of suspended particles. J. Comput. Phys. 79: 50

Ge L and Sotiropoulos F 2007 A numerical method for solving the 3d unsteady incompressible NavierStokes equations in curvilinear domains with complex immersed boundaries. J. Comput. Phys. 225(2): 1782-1809

Gilmanov A and Acharya S 2008 A hybrid immersed boundary and material point method for simulating 3d fluidstructure interaction problems. Int. J. Numer. Methods Fluids 56: 2151-2177

Goldstein D, Handler R and Sirovich L 1993 Modeling a no-slip flow boundary with an external force field. J. Comput. Phys. 105: 354-366

Huang W-X and Sung H J 2009 An immersed boundary method for fluid-flexible structure interaction. Comput. Methods Appl. Mech. Engrg. 198(33-36): 2650-2661

Iaccarino G and Verzicco R 2003 Immersed boundary technique for turbulent flow simulations. Appl. Mech. Rev. 56: 331-347

Kim D and Choi J 2000 A second-order time-accurate finite volume method for unsteady incompressible flow on hybrid unstructured grids. J. Comput. Phys. 162: 411-428

Kim J, Kim D and Choi H 2001 An immersed-boundary finite-volume method for simulations of flow in complex geometries. J. Comput. Phys. 171(1): 132-150

Kim J and Moin P 1985 Application of a fractional-step method to incompressible Navier-Stokes equations. J. Comput. Phys. 59: 308-323

Kirkpatrick M P, Armfield S W and Kent J H 2003 A representation of curved boundaries for the solution of the Navier-Stokes equations on a staggered three-dimensional Cartesian grid. J. Comput. Phys. 184: $1-36$

Labbé D F L and Wilson P A 2003 A numerical investigation of the effects of the spanwise length on the 3-d wake of a circular cylinder. J. Fluid Mech., 476: 303-334

Lai M-C and Peskin C S 2000 An immersed boundary method with formal second- order accuracy and reduced numerical viscosity. J. Comput. Phys. 160: 705-719

Li C W and Wang L L 2004 An immersed boundary finite difference method for les of flow around bluff shapes. Int. J. Numer. Methods Fluids 46: 85-107

Majumdar S, Iaccarino G and Durbin P 2001 Rans solvers with adaptive structured boundary nonconforming grids. Annual Research Briefs 353-366

Maruoka A 2003 Finite element analysis for flow around a rotating body using chimera method. Int. J. Comput. Fluid Dyn. 17: 289-297

McQueen D M and Peskin C S 1997 Shared-memory parallel vector implementation of the immersed boundary method for the computation of blood flow in the beating mammalian heart. J. Supercomput. 11: 213

Mittal R and Iaccarino G 2005 Immersed boundary methods. Annu. Rev. Fluid Mech. 37: 239-261

Mittal S and Kumar B 2003 Flow past a rotating cylinder. J. Fluid Mech., 476: 303-334

Mohd-Yosuf J 1997 Combined immersed boundary/b-spline methods for simulation of flow in complex geometries. Annual Research Briefs 317-328 
Peskin C S 1972 Flow patterns around heart valves: A numerical method. Journal of Computational Physics 10(2): 252-271

Rhie C M and Chow W L 1983 A numerical study of the turbulent flow past an isolated airfoil with trailing edge separation. AIAA J. 21: 1525-1532

Saiki E M and Biringen S 1996 Numerical simulation of a cylinder in uniform flow: Application of a virtual boundary method. J. Comput. Phys. 123: 450-465

Sohankar A, Norberg C and Davidson L 1998 Low-reynolds-number flow around a square cylinder at incidence: Study of blockage, onset of vortex shedding and outlet boundary condition. Int. J. Numer. Meth. Fluids 26: 39

Stone H L 1968 Iterative solution of implicit approximation of multidimensional partial differential equations. SIAM J. Numer. Anal. 5: 530-558

Tai C, Zhao Y and Liew K 2005 Parallel computation of unsteady incompressible viscous flows around moving rigid bodies using an immersed object method with overlapping grids. J. Comput. Phys. 207(1): $151-172$

Tau E Y 1994 A 2nd-order projection method for the incompressible Navier- Stokes equations in arbitrary domains. J. Comput. Phys. 115: 147-152

Tseng Y-H and Ferziger J H 2003 A ghost-cell immersed boundary method for flow in complex geometry. J. Comput. Phys. 192(2): 593-623

Tucker P G and Pan Z 2000 A Cartesian cut cell method for incompressible viscous flow. Appl. Math. Modell. 24: 591-606

Tyagi M and Acharya S 2005 Large eddy simulation of turbulent flows in complex and moving rigid geometries using the immersed boundary method. Int. J. Numer. Methods Fluids 48: 691-722

Unverdi S O and Tryggvason G 1992 A front-tracking method for viscous, incompressible, multi-fluid flows. J. Comput. Phys. 100: 250-37

Verma A K and Eswaran V 1999 An overlapping control volume method for Navier-Stokes equations on nonstaggered grids. Int. J. Numer. Meth. Fluids 30: 279-308

Verzicco R, Iaccarino G, Fatica M and Orlandi P 2000 Flow in an impeller stirred tank using an immersed boundary method. Annual Research Briefs 251-261

Williamson C 1988 The existence of two stages in the transition to three-dimensionality of a cylinder wake. Phys. Fluids 31: 3165-3168

Williamson C 1996 Vortex dynamics in the cylinder wake. Annu. Rev. Fluid Mech. 28: 477-539

Xia G, Zhao Y and Yeo J 2009 Parallel unstructured multigrid simulation of 3d unsteady flows and fluidstructure interaction in mechanical heart valve using immersed membrane method. Comput. Fluids 38(1): 71-79

Yang J and Stern F 2009 Sharp interface immersed-boundary/level-set method for wave-body interactions. J. Comput. Phys. 228(17): 6590-6616

Ye T, Mittal R, Udaykumar H S and Shyy W 1999 An accurate Cartesian grid method for viscous incompressible flows with complex immersed boundaries. J. Comput. Phys. 156: 209-240

Yoon D-H, Yang K-S and Choi C-B 2010 Flow past a square cylinder with an angle of incidence. Phys. Fluids 22: 043603(1-11)

Zhang S-L 1997 Gpbi-cg: Generalized product-type methods based on bi-cg for solving nonsymmetric linear systems. SIAM J. Sci. Comput. 18: 537-551

Zhang X, Ni S and He G 2008 A pressure-correction method and its applications on an unstructured chimera grid. Comput. Fluids 37: 993-1010

Zhu L and Peskin C 2003 Interaction of two filaments in a flowing soap film. Phys. Fluids 15: 128-136 